colleagues reasoned that with femtoliter chambers this would be possible, and to test this, they added a low concentration of the enzyme $\beta$-galactosidase to the chambers along with a substrate that becomes fluorescent when cleaved by the enzyme. Within 60 seconds they could see a discrete level of fluorescence in each of the chambers. When they plotted the increase in fluorescence over time for each chamber, it became apparent that each one contained zero, one, two or three enzymes. It was clear that these chambers would be ideal for studying their enzyme of choice,

$\mathrm{F}_{1}$-ATPase.

$\mathrm{F}_{1}$-ATPase is a tiny rotary enzyme, which produces ATP that serves as a major store of potential chemical energy in many biological reactions. But the efficiency with which this enzyme produces ATP from ADP has never been accurately measured. Noji and colleagues used the microchambers to confine single $\mathrm{F}_{1}$-ATPase molecules and rotated the enzyme with magnetic tweezers to produce ATP (Rondelez et al., 2005b). Because the ATP remained in the microchamber with the enzyme, once they stopped rotating the enzyme, it used the ATP to rotate in the opposite direction (Fig. 1). By comparing the number of rotations in each direction, they were able to accurately measure the efficiency of this enzyme for the very first time. This clever experiment demonstrates quite clearly the potential of these tiny 'test tubes' to examine the function of individual enzymes.

\section{Daniel Evanko}

\section{RESEARCH PAPERS}

Rondelez, Y. et al. Microfabricated arrays of femtoliter chambers allow single molecule enzymology. Nat. Biotechnol., 23, 361-365 (2005a).

Rondelez, Y. et al. Highly coupled ATP synthesis by $F_{1}$-ATPase single molecules. Nature 433, 733-777 (2005b).

tolerated in certain positions but not in others. The Cohen team incorporated these findings into computational models for prediction of miRNA targets and concluded that a large number of genes in the fly are likely to be regulated by miRNAs.

In the end, any computationally predicted mi RNA target has to be verified, and Cohen and coworkers are now testing some of their predicted targets experimentally. Their screen will tell them which of the predicted targets the miRNA actually binds to, but as Cohen points out, "It will not necessarily mean that a particular site is regulated in the natural circumstances in a cell." To prove that miRNA is working on its target in vivo, one will have to first mutate the endogenous miRNA and then demonstrate upregulation of the target gene.

Several groups are now working on such loss-of-function miRNA mutants to do proper genetic testing, whereas others are working to refine the computational prediction methods, and others still are focused on identifying more miRNA genes. It will take such multidisciplinary efforts to reveal just how influential the seemingly humble microRNA is.

\section{Nicole Rusk}

\section{RESEARCH PAPERS}

Brennecke, J. et al. Principles of microRNA-target recognition. PLoS Biology 3, e85 (2005).

\section{NEWS IN BRIEF}

\section{GENOMICS}

\section{Whole-genome patterns of common DNA variation in three human populations}

In an effort to generate a more comprehensive and relevant collection of single-nucleotide polymorphism (SNP) information, Hinds et al. conducted a detailed analysis of three distinct populations of unrelated individuals of European, African and Asian ancestry. They present the findings from their initial analysis of the extent of variation for over 1.5 million SNPs, data which they have made publicly available online. Hinds, D.A. et al. Science 307, 1072-1079 (2005).

\section{IMAGING AND VISUALIZATION}

\section{Near infrared-emissive polymersomes: self-assembled soft matter for in vivo optical imaging}

The development of a versatile and stable reagent with near-infrared fluorescence would be a considerable boon for imaging studies that require deep penetration of living tissues. Ghoroghchian et al. describe the generation of self-assembling polymersomes that incorporate an oligo(porphyrin)-based nearinfrared fluorophore and demonstrate the potential of these bodies for in vivo imaging applications.

Ghoroghchian, P.P. et al. Proc. Natl. Acad. Sci. USA 102, 2922-2927 (2005).

\section{PROTEOMICS}

\section{Interaction network containing conserved and essential protein complexes in Escherichia coli}

By adapting the yeast-based tandem affinity purification (TAP) technique for use in E. coli, Butland et al. have assembled a detailed interaction network that describes the associations between nearly 650 different bacterial proteins, confirming several predicted associations and identifing many interaction 'hubs' that seem to be broadly conserved among prokaryotes. Butland, G. et al. Nature 433, 531-537 (2005).

\section{RNA INTERFERENCE}

\section{A universal plasmid library encoding all permutations of small interfering RNA (siRNA)}

Many current RNA interference (RNAi) studies begin with a target gene and attempt to identify a suitable siRNA. Chen et al. present a tool for researchers who want to work in the reverse directiona library encoding nearly every possible 19 -nucleotide siRNA sequence- and describe how such libraries could prove valuable for conducting phenotype-driven RNA interference studies. Chen, M. et al. Proc. Natl. Acad. Sci. USA 102, 2356-2361 (2005).

\section{MICROBIOLOGY}

\section{Screening for quorum-sensing inhibitors (QSIs) by use of a new genetic system, the QSI selector}

Genes involved in quorum sensing, the process by which many bacteria organize their pathological progression in response to population density, are now recognized as an important potential antibiotic target. Rasmussen et al. demonstrate several novel genetic screens for the identification of QSIs. Rasmussen, T.B. et al. J. Bacteriol. 187, 1799-1814 (2005). 